\title{
EXPERIMENTAL DEMONSTRATION OF BEAM-BEAM COMPENSATION BY TEVATRON ELECTRON LENSES AND PROSPECTS FOR THE LHC *
}

\author{
V.Shiltsev, Y.Alexahin, V.Kamerdzhiev, G.Kuznetsov, X.L.Zhang, Fermilab, Batavia, IL, US \\ K.Bishofberger, LANL, Los-Alamos, NM, USA
}

\begin{abstract}
Electromagnetic long-range and head-on interactions of high intensity proton and antiproton beams are significant sources of beam loss and lifetime limitations in the Tevatron Collider Run II (2001-present). We present observations of the beam-beam phenomena in the Tevatron and results of relevant beam studies. We analyze the data and various methods employed in high energy physics (HEP) operation, predict the performance for planned luminosity upgrades and discuss ways to improve it.
\end{abstract}

\section{INTRODUCTION}

The luminosity of storage ring colliders is limited by the effects of electromagnetic (EM) interaction of one beam on the particles of the other beam which leads to a blowup of beam sizes, a loss of beam intensities and unacceptable background rates in the high energy physics (HEP) detectors. This beam-beam interaction is parameterized by a dimensionless beam-beam parameter $\xi \equiv r_{0} N / 4 \pi \varepsilon$, where $r_{0}=e^{2} / m c^{2}$ denotes the particle's classical radius, $N$ is the opposing bunch's intensity and $\varepsilon$ is its the rms normalized emittance related to transverse rms beam size $\sigma$ at the interaction point (IP) as $\varepsilon=\gamma \sigma^{2} / \beta^{*}, \gamma$ is relativistic gamma factor, $\beta^{*}$ is the betafunction at the IP (for simplicity here, we consider a collider with round Gaussian beams). This dimensionless parameter is equal to the shift of the betatron oscillation tune of core particles due to beam-beam forces. While core particles undergo a significant tune shift, halo particles with large oscillation amplitudes experience negligible tune shift. The EM forces drive nonlinear resonances which can result in particle instability and loss. The beam-beam limit in modern hadron colliders is at $\xi^{\max } \approx 0.005-0.01$ per IP, while it can exceed $\xi^{\max } \approx 0.1$ per IP in high energy electron-positron colliders [1].

Operation with a greater number of bunches allows a proportional increase of luminosity but requires careful spatial separation of two beams everywhere except at the main IPs. Long-range (as opposed to head-on) EM interactions of separated beams are also nonlinear and contribute to the limit on collider performance. These long-range effects usually vary from bunch to bunch, making their treatment even more cumbersome.

Besides the technique of electron lenses, the subject of

\footnotetext{
*Work supported by Fermi Research Alliance Ltd. under Contract No. DE-AC02-76CH03000 with the US DoE

"shiltsev@fnal.gov
}

this report, there are few beam-beam compensation (BBC) schemes tested experimentally. The $0.8-\mathrm{GeV}$ DCI storage ring at the Laboratoire de l'Accelerateur Lineaire (Orsay, France) had four colliding beams - one positron and one electron coming from each direction. Full space charge and current compensation could be achieved if all the beams had the same intensities and dimensions, but the observed beam-beam limit was not significantly different than with just two beams [2]. These results are attributed to strong coherent beam-beam effects which are characterized by rapid correlated variations of the beam distributions - see Ref.[3] and references therein. Octupole magnets were used for compensation of the cubic nonlinearity in the beam-beam force at the VEPP-4 $e+e$ - collider (Novosibirsk, Russia) [4]. Although a several-fold reduction of electron halo loss rate was demonstrated at optimal octupole current, the technique has not found wide application because its efficiency is strongly dependent on the machine tune. Compensation of the EM fields of separated beams by placing a current conducting wire at the same distance to the beam as opposite beam was proposed in [5]. Some $20 \%$ reduction of the $e+$ loss rate due to such a method was observed at the DAFNE (Frascatti, Italy) [6]. The wire-compensation technique is less efficient if multiple beam-beam interactions occur at different distances and betatron phases, and, of course, it is useless for head-on BBC.

\section{TEVATRON ELECTRON LENSES}

Electron lenses were proposed for compensation of both long-range and head-on beam-beam effects in the Fermilab's Tevatron collider (Batavia, USA) [7]. The lens employs a low energy $\beta_{e}=v / c<<1$ beam of electrons which collides with the high-energy bunches over an extended length $L_{e}$. Electron space charge forces are linear at distances smaller than the characteristic beam radius $r<a$ but scale as $1 / r$ for $r>a$.

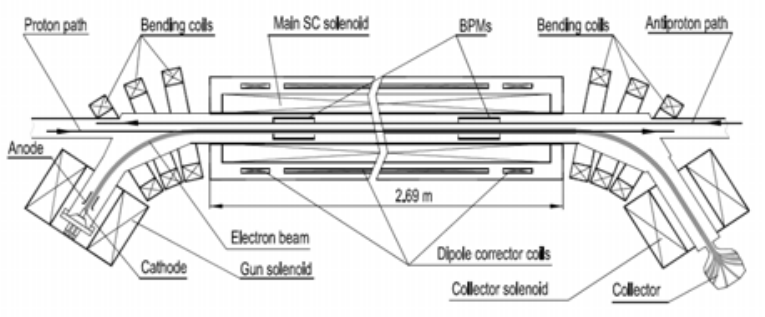

Figure 1: Layout of the Tevatron Electron Lens.

Correspondingly, such a lens can be used for linear and nonlinear force compensation depending on the beam-size ratio $a / \sigma$ and the current-density distribution $j(r)$. Main 
advantages of the electron lens compensation are: a) the electron beam acts on high-energy beams only through EM forces (no nuclear interaction), eliminating radiation issues; b) fresh electrons interact with the high-energy particles each turn, leaving no possibility for coherent instabilities; c) the electron current profile (and thus the EM field profiles) can easily be changed for different applications; d) the electron-beam current can be adjusted between each of the bunches, equalizing the bunch-tobunch differences and optimizing the performance of all of the bunches in multi-bunch colliders.

Two Tevatron Electron Lenses (TELs) were built and installed in two different locations of the Tevatron ring, A11 and F48. Fig.1 depicts a general layout of the TELs. The TEL and Tevatron parameters are given below.

\begin{tabular}{llll}
\hline Parameter & Symbol & Value & Unit \\
& Tevatron Electron Lens & \\
Electron beam energy & $U_{e}$ & $5 / 10(\max )$ & $\mathrm{kV}$ \\
Peak electron current & $J_{e}$ & $0.6 / 2.3(\max )$ & $\mathrm{A}$ \\
Main/gun solenoid field & $B_{\text {main }} / B_{\text {gun }}$ & $30.1 / 2.9$ & $\mathrm{kG}$ \\
Radii: cathode/e-beam & $a_{c} / a_{e}$ & $7.5 / 2.3$ & $\mathrm{~mm}$ \\
Radii: cathode radius & $a_{c}$ & 7.5 & $\mathrm{~mm}$ \\
e-pulse period/width & $T_{0} / T_{e}$ & $21 / \approx 0.6$ & $\mu \mathrm{s}$ \\
length & $L_{e}$ & 2.0 & $\mathrm{~m}$ \\
& $C_{\text {Tevatron }}$ Collider Parameters & \\
Circumference & $C$ & 6.28 & $\mathrm{~km}$ \\
p/pbarbeam energy & $E$ & 980 & $\mathrm{GeV}$ \\
p/pbar bunch intensity & $N_{p} / N_{a}$ & $\approx 250 / 50-100$ & $10^{9}$ \\
Emittance p/pbar(rms) & $\varepsilon_{p} / \varepsilon_{a}$ & $\approx 2.8 / 1.4$ & $\mu \mathrm{m}$ \\
\#bunches/bunch spacing & $N_{B} / T_{b}$ & $36 / 396$ & $\mathrm{~ns}$ \\
Initial luminosity & $L_{0}$ & $1.5-2.910^{32}$ & $\mathrm{~cm}{ }^{-2} \mathrm{~s}^{-1}$ \\
$B$-functions at A11 (F48) & $\beta_{y} / \beta_{x}$ & $150 / 68(29 / 104)$ & $\mathrm{m}$ \\
p/pbar head-on tuneshift & $\xi^{\mathrm{p}} / \xi^{\mathrm{a}}$ & $\approx 0.008 / 0.011$ & $\mathrm{per} \mathrm{IP}$ \\
P/pbar long-range $d Q$ & $\Delta Q^{p} / \Delta Q^{a}$ & $\approx 0.003 / 0.006$ & $\mathrm{max}$.
\end{tabular}

In order to keep electron beam straight and its distribution unaffected by its own space-charge and main beam EM fields, the electron beam is immersed in a strong magnetic field - about $3 \mathrm{kG}$ at the electron-gun cathode and some $30 \mathrm{kG}$ inside the main superconducting (SC) solenoid. The deviations of the magnetic field lines from a straight line are less than $\pm 100 \mu \mathrm{m}$ over the entire length of the SC solenoid. The electron beam, following the field lines, therefore does not deviate from the straight Tevatron beam trajectory by more than $20 \%$ of the Tevatron beam rms size $\sigma \approx 0.5-0.7 \mathrm{~mm}$ in the location of the TELs.

The electron beam's transverse alignment on the proton or antiproton bunches (within $0.2-0.5 \mathrm{~mm}$ all along the interaction length) is crucial for successful BBC. The electron beam steering is done by adjusting currents in superconducting dipole correctors installed inside the main solenoid cryostat. It was also important that electron gun generates electron current distribution with wide flat top and smooth radial edges. Such a distribution is generated in the 7.5 -mm radius convex cathode electron gun with an optimized electrode geometry [8]. The TEL magnetic system compresses the electron-beam crosssection area in the interaction region by the factor of $B_{\text {main }}$ $B_{\text {gun }} \sim 10$ (variable from 2 to 30 ), proportionally increasing the current density of the electron beam in the interaction region. Most current experiments have not required more than $0.6 \mathrm{~A}$, though previous tests up to $3.0 \mathrm{~A}$ have been performed. In order to enable operation on a single bunch in the Tevatron with bunch spacing of $396 \mathrm{~ns}$, the anode voltage, and consequently the beam current, are modulated with a characteristic on-off time of about $0.6 \mu \mathrm{s}$ and a repetition rate equal to the Tevatron revolution frequency of $f \_0=47.7 \mathrm{kHz}$ by using a HV Marx pulse generator or a HV RF tube base amplifier. The electron pulse timing jitter is less than $1 \mathrm{~ns}$ and the peak current is stable to better than $1 \%$, so, the TEL operation does not incur any significant emittance growth. Detailed description of the TEL is given in Ref. [9] and references therein.

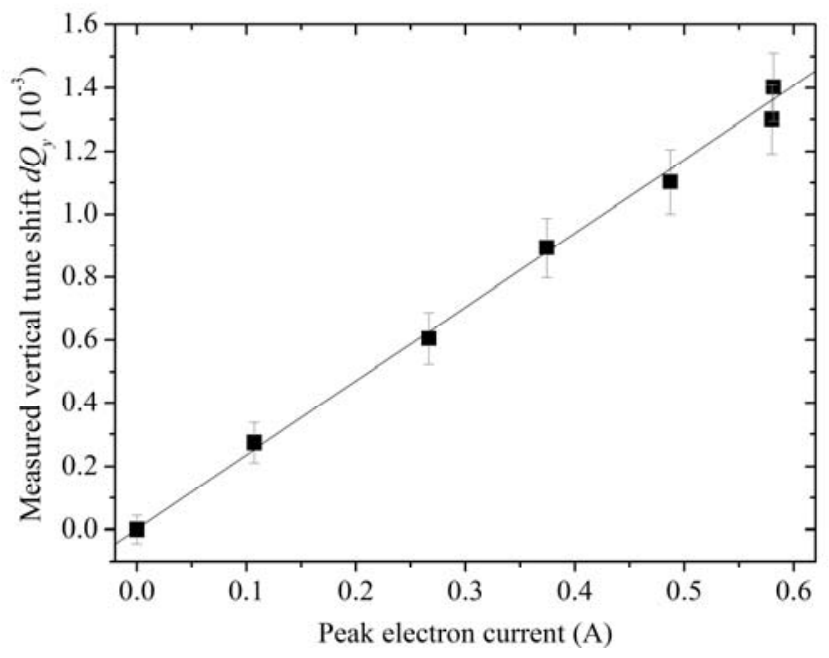

Figure 2: Vertical betatron tune shift of $980-\mathrm{GeV}$ proton bunch vs. the peak electron current in the A11 TEL

The high-energy protons are focused by the TEL and experience a positive betatron tune shift given by [7]:

$$
d Q_{x, y}=+\frac{\beta_{x, y} L_{e} r_{p}}{2 \gamma e c} \cdot j_{e} \cdot\left(\frac{1-\beta_{e}}{\beta_{e}}\right)
$$

The tune shift is about the same for most protons in the bunch since $a \sim 3 \sigma$. Fig. 2 presents results of the measurements of the vertical tune shift $d Q_{y}$ of $980-\mathrm{GeV}$ protons versus electron current in the TEL installed at the A11 location with a vertical beta-function of $\beta_{y}=150 \mathrm{~m}$, in good agreement with Eq.(1) using the values of $\beta_{e}=0.14$ for $5-\mathrm{keV}$ electron energy and $j_{e} \equiv J_{e} / \pi a_{e}{ }^{2}=0.05 \mathrm{~A} / \mathrm{mm}^{2}$ for a 0.6-A beam with an effective radius of about 2 millimeters - see solid line. 


\section{BEAM-BEAM COMPENSATION}

\section{$B B C$ by vertical electron lens}

One of the most detrimental effects of the beam-beam interaction in the Tevatron is the significant attrition rate of protons due to their interaction with the antiproton bunches in the main IPs (B0 and D0) and due to numerous long-range interactions [10]. The effect is especially large at the beginning of the HEP stores where the positive proton tune shift due to focusing by antiprotons at the main IPs can reach $\xi=0.016$. Fig. 3 shows a typical distribution of proton loss rates at the beginning of an HEP store. In the Tevatron, 36 bunches in each beam are arranged in 3 trains of 12 bunches separated by $2.6 \mu \mathrm{s}$ long abort gaps. Proton bunches \#12, 24, and 36 at the end of each bunch train typically lose about $9 \%$ of their intensity per hour while other bunches lose only (4-6)\% $/ \mathrm{hr}$. These losses are a very significant part of the total luminosity decay rate of about $20 \%$ per hour (again, at the beginning of the high luminosity stores). The losses due to inelastic proton-antiproton interactions at the two main IPs are much smaller (1.1-1.5\%/hr). Fig. 3 shows large bunch-to-bunch variations in the beam-beam induced proton losses within each bunch train but similar rates for equivalent bunches, e.g. \#12, 24, and 36 .

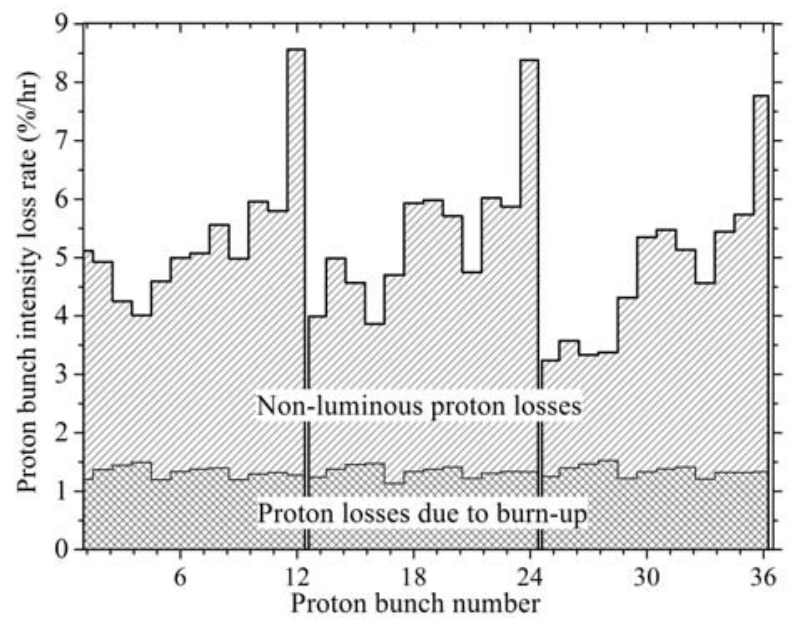

Figure 3: Proton-bunch intensity loss rates at the beginning of the Tevatron store \#5155, Dec. 30, 2006, with initial luminosity $2.5 \cdot 10^{32} \mathrm{~cm}^{-2} \mathrm{~s}^{-1}$.

In the $\mathrm{BBC}$ demonstration experiment, we centered and timed the electron beam of the A11 TEL onto bunch \#12 without affecting any other bunches. When the TEL peak current was increased to $J=0.6 \mathrm{~A}$, the lifetime $\tau=N /(d N / d t)$ of bunch \#12 went up to 26.6 hours from about 12 hours see Fig.4. At the same time, the lifetime of bunch \#36, an equivalent bunch in the third bunch train, remained low and did not change significantly (at 13.4 hours lifetime). When the TEL current was turned off for fifteen minutes, the lifetimes of both bunches were, as expected, nearly identical (16 hours). The TEL was then turned on again, and once again the lifetime for bunch \#12 improved significantly to 43 hours while bunch \#36 stayed poor at 23.5 hours. This experiment demonstrates a factor of two improvement in the proton lifetime due to compensation of beam-beam effects with the TEL.

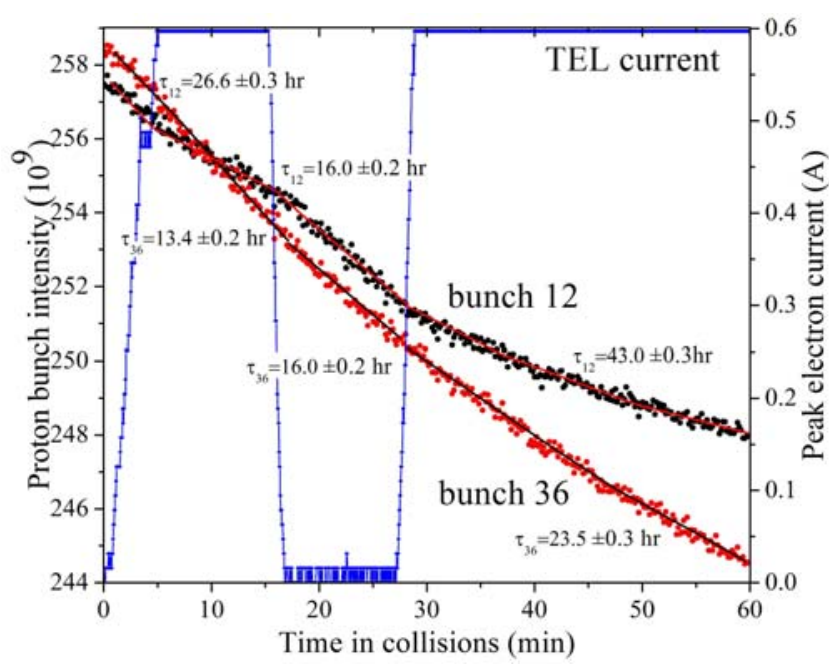

Figure 4: Proton-bunch intensity loss rates at the beginning of the Tevatron store \#5119, Dec. 30, 2006, with initial luminosity $2.5 \cdot 10^{32} \mathrm{~cm}^{-2} \mathrm{~s}^{-1}$.

The proton lifetime, dominated by beam-beam effects, gradually improves and reaches roughly 100 hours after 6-8 hours of collisions; this is explained by a decrease in antiproton population and an increase in antiproton emittance, both contributing to a reduction of the proton beam-beam parameter $\xi$. To study the effectiveness of $\mathrm{BBC}$ later in the store, the TEL was repeatedly turned on and off every half hour for 16 hours, again on bunch \#12. The relative improvement $R$, defined as the ratio of the proton lifetime with the TEL and without, is plotted in Fig.5.

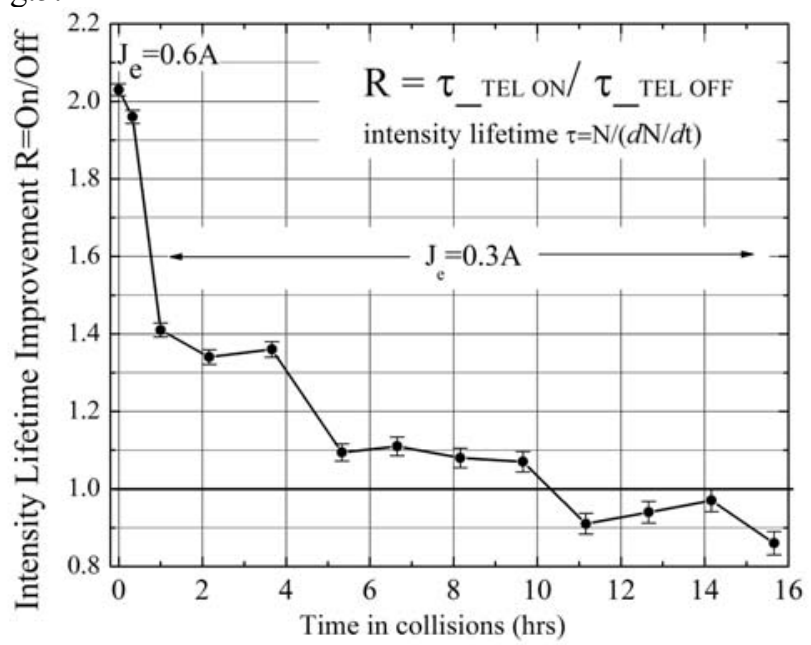

Figure 5: Relative improvement of the TEL induced proton bunch \#12 lifetime vs. time (store \#5119).

The first two data points correspond to $J=0.6 \mathrm{~A}$ (as is Fig. 4 and the above description), but subsequent points were taken with $J=0.3 \mathrm{~A}$ to observe dependence of the compensation effect on electron current. The change of the current resulted in a drop of the relative improvement from $R=2.03$ to $R=1.4$. A gradual decrease in the relative 
improvement is visible until after about ten hours, where the ratio reaches 1.0 (no gain in lifetime). At this point, the beam-beam effects have become very small, providing little to compensate. Similar experiments in several other stores with initial luminosities ranging from $1.5 \cdot 10^{32} \mathrm{~cm}^{-2}$ $\mathrm{s}^{-1}$ to $2.5 \cdot 10^{32} \mathrm{~cm}^{-2} \mathrm{~s}^{-1}$ repeated these results.

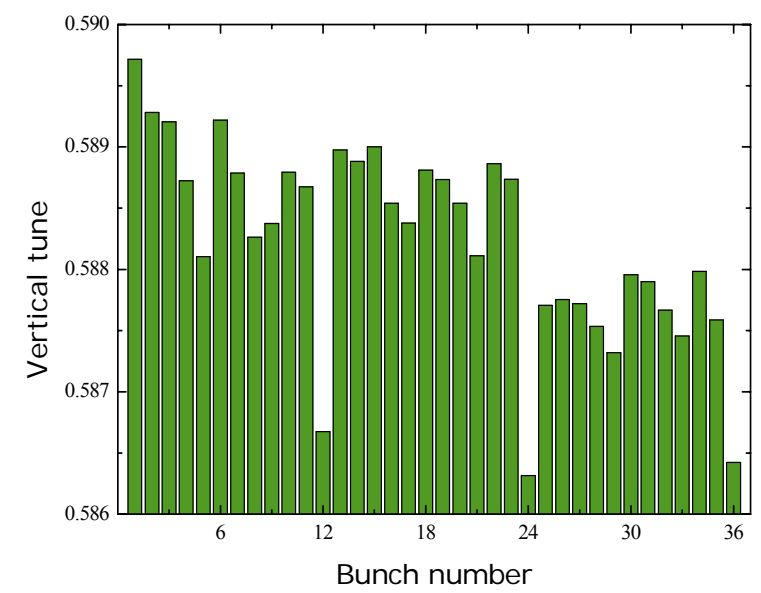

Figure 6: Vertical tunes of proton bunches measured by DTM at the beginning of store \#5301, Mar. 27, 2007 with initial luminosity $2.5 \cdot 10^{32} \mathrm{~cm}^{-2} \mathrm{~s}^{-1}$

The lifetime improvement due to the TEL can be explained in part by the positive shift of vertical tune of protons $d Q_{y} \approx 0.0015$ which makes the detrimental effects of the $12^{\text {th }}$ order resonance $Q_{y}=7 / 12=0.583$ weaker. The average Tevatron proton tune $Q_{y}=0.589$ (which is carefully optimized to minimize overall losses) is just above this resonance, and the bunches at the end of each train, which have vertical tunes lower by $\Delta Q_{y}=-(0.002-$ 0.003) due to a unique schedule of long range beambeam interactions, are subject to stronger beam-beam effects [10]. Vertical proton tunes measured by Digital Tune Meter [11] at the start of a store are shown in Fig.6.

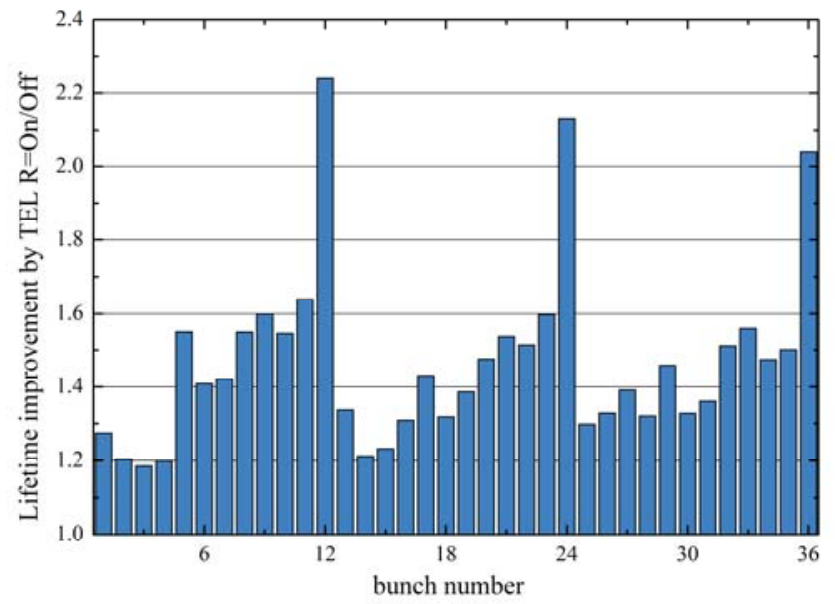

Figure 7: Proton bunch lifetime improvements due to $J=0.3 \mathrm{~A}$ in TEL-2 at the beginning of store \#5301.
The TEL moves those protons away from the resonance, thus, resulting in significant reduction of the losses. It is noteworthy, that the TEL operation with $J=0.3$ A resulted in bunch $\# 12$ having one of the lowest loss rates among all bunches, while its tune still remained lower $d Q_{y}<\left|\Delta Q_{y}\right|$.

Fig.7 shows that the relative lifetime improvement (TEL-2 with $J=0.3 \mathrm{~A}$ of DC on vs TEL-2 off) can reach $R=2.2$ for some bunches while the tune shift is not that big $(\sim 0.0008)$. In that particular store \#5301 we turned TEL2 off and on regularly and compared the average lifetime improvement factor due to TEL-2 with the improvement due to intentional equivalent tune change. As Figs. 8 and 9 show, the effects on intensity lifetime were comparable, while luminosity lifetime improvement due to TEL-2 was somewhat bigger than due to the $d Q y$ change $(\sim 12 \%$ at the beginning of the store).

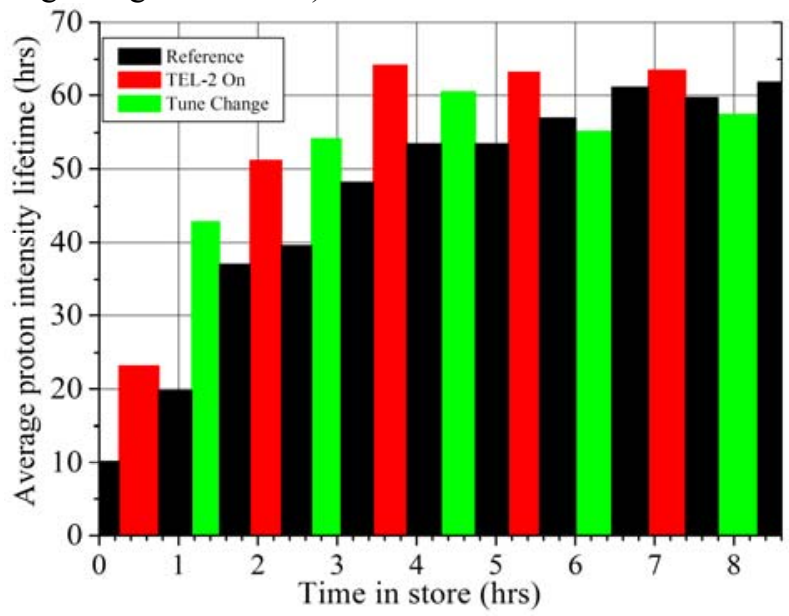

Figure 8: Average proton lifetime in store \#5301, redTEL on with $J=0.3 \mathrm{~A}$, green - vertical protyon tune bumped up 0.0008 , black bars - no tune change and no TEL-2 current

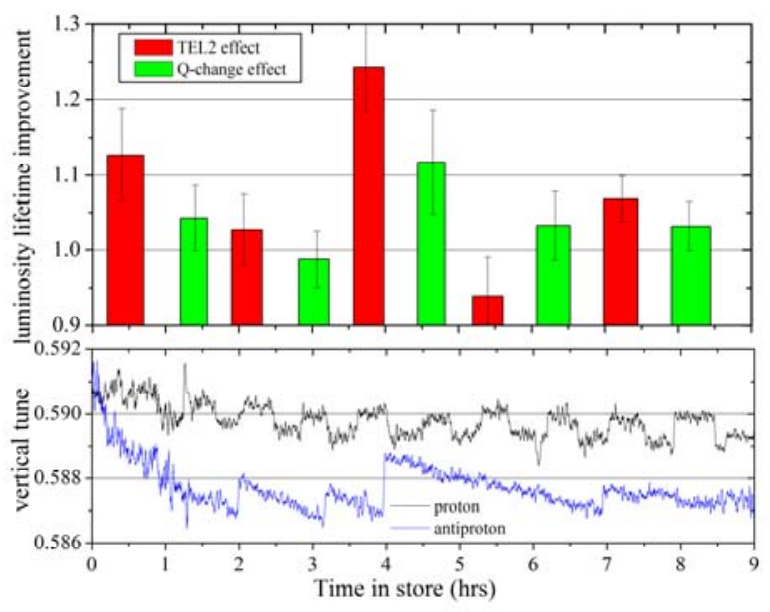

Figure 9: Improvement of luminosity lifetime by TEL1 (red) and due to 0.0008 vertical tune change (green). Measured proton vertical and horizontal plots are shown in the lower plot. 


\section{$B B C$ by horizontal electron lens}

TEL-1 is located in the location with large horizontal beta-function and mostly shifts horizontal proton tune up. Fig. 10 below shows that horizontal lens improved proton lifetime as well (by about 40\%). Fig.11 demonstrates that if electron beam is displaced from proton orbit by more than $4 \mathrm{~mm}$, then the effect of the TEL-1 vanished.

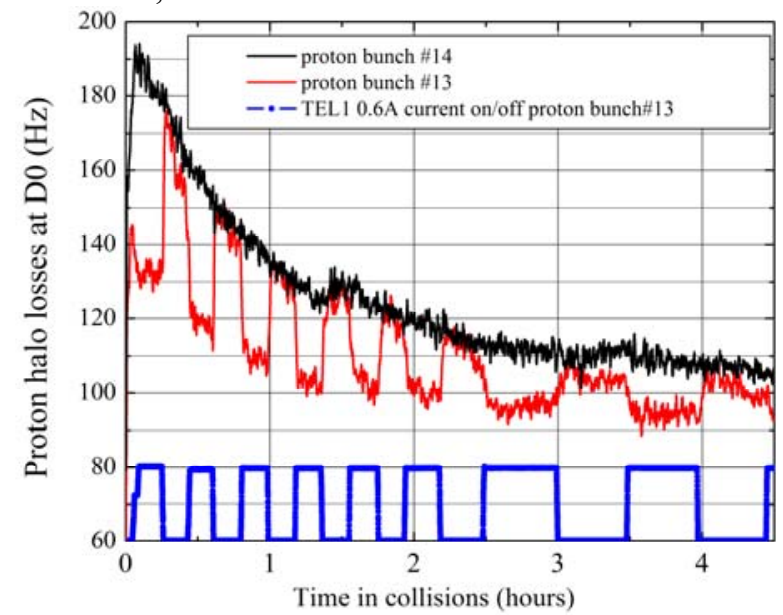

Figure 10: Losses from proton bunches \#13 and \#14 at the beginning of the HEP store \#5352, Apr. 13, 2007 with initial luminosity $1.97 \cdot 10^{32} \mathrm{~cm}^{-2} \mathrm{~s}^{-1}$. TEL-1 with $0.6 \mathrm{~A}$ of current intermittently acted on bunch \#13 only.

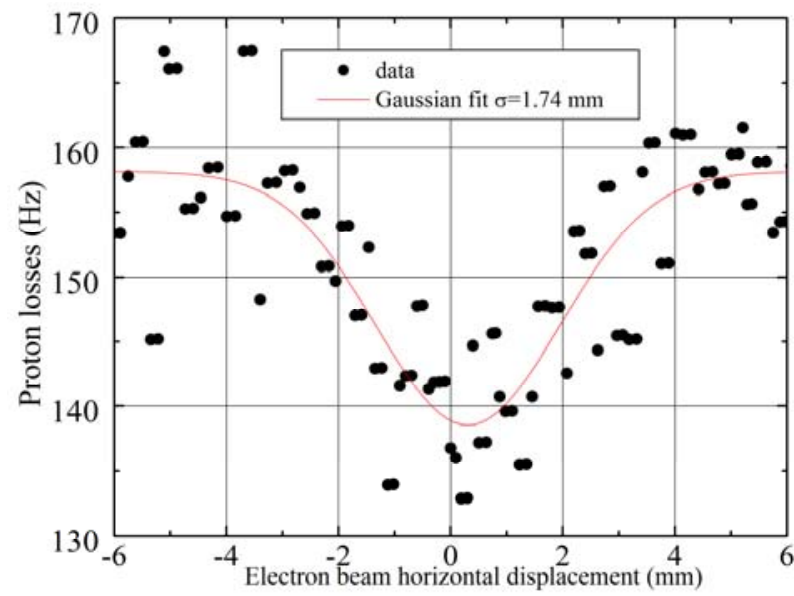

Figure 11: Dependence of proton losses on horizontal position of TEL-1 electron beam.

\section{BEAM-BEAM COMPENSATION IN LHC}

The versatility of electron lenses allows their use in many other applications, also. For example, the TEL installed at F48 location in the Tevatron for several years is used for removing unwanted DC beam particles out of the Tevatron abort gaps between the bunch trains [12]. Several other electron lens concepts have been proposed for space-charge compensation in high intensity proton synchrotrons [13], reduction of a tune spread in protonproton or like-charge colliding beams [14,7], and beam collimation in the LHC [15]. An LHC electron lens with about 2.4 A of DC current can compensate head-on effects induced by collisions with $2.3 \mathrm{e} 11$ proton bunches (twice the LHC nominal bunbch intensity) - see Fig.12 from [16]. As such, the electron lenses combined with current carrying wires for long-range beam-beam compensation are believed to allow to reach higher collider luminosities without significant increase of particle loss rates or emittance growth rates.

Figure 12: LHC footprint reduction by electron lens for
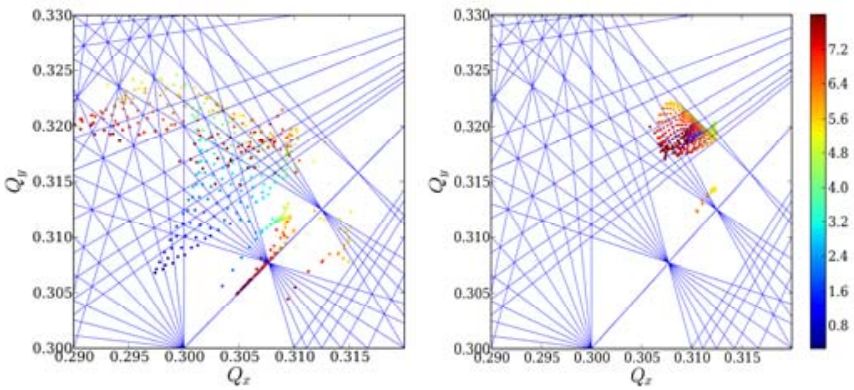

full head on compensation by electron lenses and longrange compebnsation by wires. Left plot - the LHC with beams with $N p=2.3 \mathrm{e} 11 /$ bunch and no e-lens, right - with beam-beam compensation (courtesy of U.Dorda).

We would like to thank our colleagues from FNAL, BINP, IHEP and CERN for their help, technical contributions and fruitful discussions.

\section{REFERENCES}

[1] W.Fischer and T.Sen, in Proceedings of HALO-2003 Workshop,. J.Wei, W.Fischer, P.Manning, eds, AIP Conf. Proc. No. 693 (AIP, New York, 2003), p.215.

[2] J.LeDuff et al ., in Proc. $11^{\text {th }}$ Int. Conf. on HighEnergy Accel., ed. W.S.Newman (1980), p.707.

[3] B.Podobedov, R.H.Siemann, Phys. Rev. E52, p. 3066 (1995).

[4] A.Temnykh, in Proc. $3^{\text {rd }}$ Advanced ICFA BD Workshop on Beam-Beam Effects in Circular Colliders, I.Koop, G.Tumaikin, eds., 1989 (Novosibirsk), p.5

[5] J.P.Koutchouk, in Proc. PAC'2001, Chicago, p.1681.

[6] M.Zobov et al., in Proc. EPAC'06, Edinburg, p.2808.

[7] V.Shiltsev et al., Phys. Rev. STAB 2, 071001 (1999).

[8] V.Kamerdzhiev et al., in Proc. $12^{\text {th }}$ Advanced Accelerator Concepts Workshop, AIP Conf. Proc. No. 877 (AIP, New York, 2006), pp.609-615.

[9] K.Bishofberger, Ph.D. Thesis, UCLA(2005); see also V.Kamerdzhiev et al. these Proceedings

[10] V.Shiltsev et al., Phys. Rev. STAB 8, 101001 (2005); see also A.Valishev, et al, these Proceedings.

[11] V.Kamerdzhiev, et al, these Proceedings.

[12] X.L.Zhang et al., in Proc. PAC'0, Portland, p.1778.

[13] A.Burov et al., Fermilab TM-2125 (2000).

[14] E.Tsyganov et al., Fiz. Elem. Chastits At.Yad. 27, 675 (1996) [Phys. Part. Nuclei 27, 279 (1996)].

[15] V.Shiltsev, Fermilab Conf-06-505 (2006).

[16] U.Dorda, et al, these Proceedings. 
